\title{
Tecniche non invasive di riposizionamento del catetere per dialisi peritoneale
}

$A$ più di venti anni dalla sua introduzione, il catetere peritoneale di Tenckhoff nella sua versione originale è ancora certamente il tipo più largamente utilizzato al mondo.

Una delle complicanze tecniche di più frequente riscontro in CAPD è l'interruzione del flusso in uscita e/o in entrata del liquido peritoneale dovuta a migrazione o incarceramento da parte dell'omento, della punta pelvica del catetere $(1,2)$.

Una corretta tecnica di inserimento può ridurre la frequenza di migrazione del catetere, ma non elimina il problema che in alcune casistiche selezionate arriva a interessare più del $10 \%$ dei cateteri inseriti $(3$, 4). Lo stesso Tenckhoff raccomandava un inserimento profondo nella pelvivera che avrebbe messo al riparo la punta del catetere dalla trappola dell'omento che non raggiunge mai quella sede anatomica. Inoltre consigliava l'inserimento in direzione caudale della porzione intramurale $\mathrm{e}$ il confezionamento del tunnel sulla destra e dell'"exitsite" rivolto verso l'alto. Tutto ciò per evitare che le forze peristaltiche

\author{
R. Galato \\ Divisione di Nefrologia e Dialisi
Ospedale Cà Granda - Milano \\ Divisione di Nefrologia e Dialisi
Ospedale Cà Granda - Milano
}

del colon ascendente influenzassero in maniera negativa la posizione del "tip" e nello stesso tempo perché così facendo si preservava la forma naturale retta del catetere; ogni cambiamento, infatti, della forma del catetere avrebbe generato, per una sorta di "memoria della forma", forze capaci di promuovere la migrazione dello stesso (5).

Pur rispettando questi avveduti consigli, la frequenza di migrazione e il relativo malfunzionamento idraulico del catetere rimangono elevati e pongono problemi di strategia operativa. Attendere con

provvedimenti conservativi una risoluzione spontanea, sperabile quando la migrazione avvenga in ipocondrio sinistro, oppure aggredire chirurgicamente il catetere nel caso di migrazione sotto epatica? Noi in ogni caso proponiamo un semplice ed efficace metodo di rilocazione del catetere peritoneale con tecnica non invasiva. Riportiamo la nostra esperienza di cinque anni dell'uso di un semplice ed efficace metodo di rilocazione del catetere peritoneale con tecnica non invasiva da noi già descritta (6). 27 pazienti, 15 maschi e 12 femmi-
Fig. 1 - Un catetere di Fogarty (PVC Modello 32-08-6F) è in grado di essere introdotto lungo il catetere di Tenckhoff e di superare l'estremità interna di alcuni centimetri. Dopo l'inserimento il palloncino viene quindi riempito con mercurio ed il catetere può essere fissato all'estremità esterna del Tenckhoff e lasciato in sede per 24 ore.

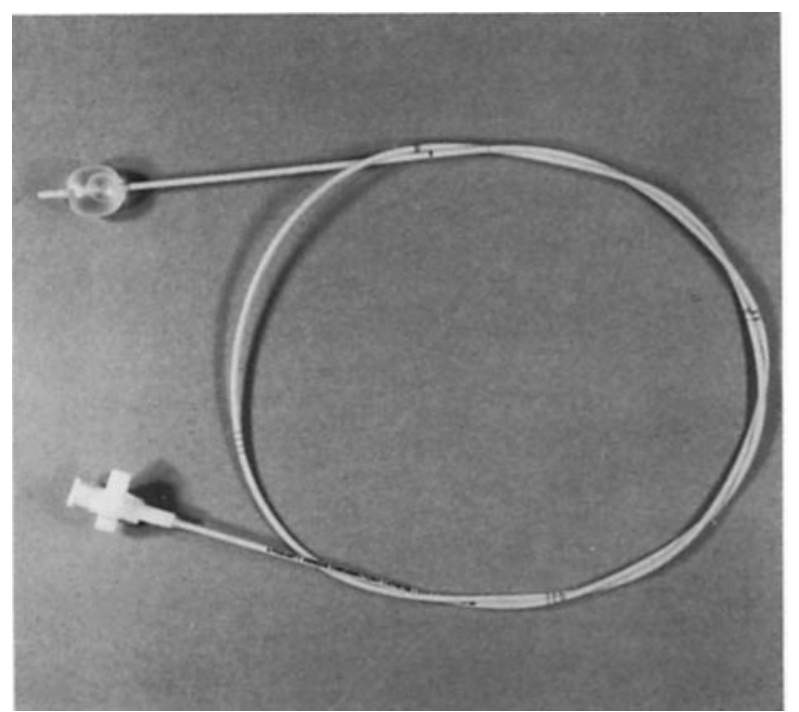




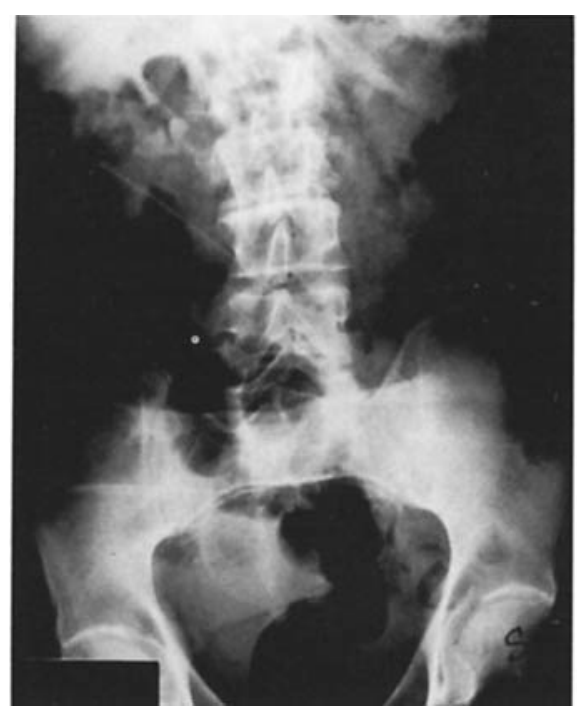

Fig. 2A - Esempio di dislocazione di catetere peritoneale in ipocondrio destro.

ne, di età compresa tra 59 e 77 anni, in CAPD da 2 a 22 mesi, portatori di catetere di Tenckhoff a due cuffie inserito sulla linea mediana $2 \mathrm{~cm}$ sotto l'ombelico, hanno improvvisamente presentato interruzione del normale drenaggio del liquido peritoneale. Uno studio radiografico dell'addome senza mezzo di contrasto dimostrava migrazione del catetere peritoneale in 11 casi in ipocondrio destro, in 7 casi in ipocondrio sinistro, in 6 casi in epigastrio e in 3 casi in fianco destro.

Le usuali manovre messe in atto per ripristinare la funzione idraulica del catetere come la deambulazione prolungata, i purganti, il flusso forzato di liquido attraverso il catetere, non sortivano alcun effetto.

Attraverso il catetere di Tenckhoff, in condizioni di asepsi, abbiamo allora introdotto in cavità peritoneale un catetere Fogarty (PVC Model 32-08-6F) superandone col palloncino l'estremità interna di 1 $\mathrm{cm}$. Il palloncino è stato quindi riempito con $1 \mathrm{cc}$. di mercurio metallico ed il catetere Fogarty è stato fissato a tenuta all'estremità esterna del catetere peritoneale e lascia-

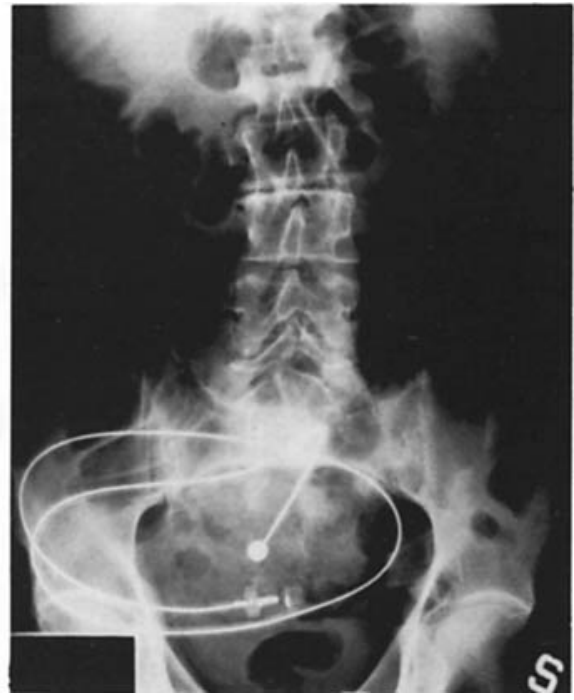

Fig. 2B - Il catetere viene a collocarsi in sede 24 ore dopo l'inserimento del catetere di Fogarty appesantito con mercurio nel palloncino.

to in sede per 24 ore.

Dopo tale periodo di stazionamento i controlli radiografici dimostravano in 25 casi $(92.5 \%)$ una ridiscesa della punta del catetere in pelvi. All'estrazione del catetere Fogarty la dialisi peritoneale ha potuto regolarmente riprendere in 25 casi dei 27 cosi trattati e solo in 2 casi si è reso necessario un riposizionamento chirurgico. Non si è mai avuto alcuno spandimento di mercurio nella cavità, tenuto conto che il riempimento del palloncino del Fogarty è assai al disotto della capacità consentita.

Il catetere di Tenckhoff classico rimane il catetere di più semplice inserimento e quindi è ancora il catetere più usato pur presentando una relativamente elevata incidenza di migrazione.

La possibilità di riposizionare con facilità, efficacia, sicurezza e senza intervento chirurgico il catetere dislocato rende meno importante tale complicanza (7).

Riteniamo quindi che la tecnica da noi proposta sia da utilizzare come prima scelta in caso di dislocazione del catetere peritoneale.

\section{Bibliografia}

1. Glor HJ, Nikols K, Sorkin M et al. Peritoneal access and related complication in CAPD. Am J Med 1983; 74: 593-8.

2. Ponce SP, Pierratos $A$, Izzat $S$ et al. Comparison of the survival and complications of three permanent peritoneal dialysis catheters. Perit Dial Bull 1982; 2: 82-5.

3. Robinson RG, Leapman SB et al. Surgical considerations of continuous ambulatory peritoneal dialysis. Surgery 1984; 723.

4. Olcott C IV, Feldman CA, Coplon NS et al. CAPD technique of catheter insertion and management of associated surgical complications. Am J Surg 1983; 246: 98-102.

5. Tenckhoff H. Peritoneal dialysis today. A new look. Nephron $1974 ; 19: 67$.

6. Galato R, Seveso M, Perego A et al. Riposizionamento del catetere peritoneale senza intervento chirurgico. Atti del III $^{\circ}$ Convegno Nazionale sulla Dialisi Peritoneale, S. Margherita Ligure. Milano: Wichtig Ed. 1985; 301-2.

7. Korten G, Arendt R, Brugman E et al. Relocation of a peritoneal catheter without surgical intervention. Perit Dial Bull 1983; $1: 46$. 